03,06

\title{
Генерация второй оптической гармоники и ее фотоиндуцированная динамика в сегнетоэлектрике-полупроводнике $\mathrm{Sn}_{2} \mathbf{P}_{2} \mathbf{S}_{6}$
}

\author{
(C) К.А. Брехов ${ }^{1}$, К.А. Гришунин ${ }^{1}$, Д.В. Афранасьев ${ }^{2}$, С.В. Семин ${ }^{1,2}$, \\ Н.Э. Шерстюк ${ }^{1}$, Е.Д. Мишина ${ }^{1}$, А.В. Кимель ${ }^{1,2}$ \\ ${ }^{1}$ Московский технологический университет (МИРЭА), \\ Москва, Россия \\ ${ }^{2}$ Radboud University, Institute for Molecules and Materials, \\ Nijmegen, The Netherlands \\ E-mail: brekhov_ka@mail.ru \\ (Поступила в Редакцию 31 мая 2017 г.)
}

Методом оптической накачки-зондирования исследована сверхбыстрая динамика нелинейно-оптического отклика кристалла сегнетоэлектрика-полупроводника $\mathrm{Sn}_{2} \mathrm{P}_{2} \mathrm{~S}_{6}$, возбуждаемая с помощью фемтосекундного лазерного импульса. Показано, что под воздействием фемтосекундных импульсов в образце происходит изменение генерации второй оптической гармоники, что может быть связано с экранировкой существующей электрической поляризации.

Работа выполнена при финансовой поддержке Министерства образования и науки РФ (грант 14.Z50.31.0034, госзадание 3.7500.2017/9.10).

DOI: 10.21883/FTT.2018.01.45285.179

\section{1. Введение}

На пути к полному оптическому переключению намагниченности в магнитоупорядоченных средах был пройден длинный путь. Первые исследования, посвященные этой тематике, показали, что возбуждение лазерным импульсом длительностью $60 \mathrm{fs}$ разрушает магнитный порядок металлической пленки ферромагнитного никеля на временах порядка 1 ps [1]. После этого была продемонстрирована возможность возбуждения светом колебаний спинов на частоте магнитного резонанса [2-4]. И хотя изначально амплитуды этих колебаний не превышали нескольких градусов, в дальнейшем было показано, что вблизи фазового перехода эти амплитуды могут быть заметно усилены [5]. В работах [6] и [7] была продемонстрирована возможность переориентации спинов на 90 и $180^{\circ}$ соответственно под действием фемтосекундного лазерного импульса. С учетом этих результатов была показана возможность оптической записи информации с помощью короткого лазерного импульса и за предельно короткое время [8]. Наконец, в работах [9-11] была продемонстрирована возможность записи информации путем воздействия фемтосекундных лазерных импульсов на магнитный носитель.

Важно отметить, что, несмотря на очевидные аналогии между магнитоупорядоченными и сегнетоэлектрическими материалами, возможность сверхбыстрого переключения параметра порядка в сегнетоэлектриках изучена крайне мало и продолжает оставаться актуальной задачей, решение которой позволит создать новые более эффективные устройства сегнетоэлектрической памяти [12].

Ранее [13] нами была продемонстрирована возможность возбуждения мягкой фононной моды в кристал- ле сегнетоэлектрика-полупроводника $\mathrm{Sn}_{2} \mathrm{P}_{2} \mathrm{~S}_{6}$ (SPS) под действием фемтосекундного лазерного импульса. Это может свидетельствовать о возможности влияния света на сегнетоэлектрический параметр порядка и, по аналогии с магнитными материалами, может стать первым шагом на пути к полному оптическому переключению сегнетоэлектрической поляризации.

Традиционным методом детектирования фазового перехода является метод рентгеновской дифракции. Однако эта методика довольно сложна для исследования фазового перехода с временным разрешением [14].

Методика генерации второй оптической гармоники (ГВГ) уже давно является одним из основных методов исследования свойств сегнетоэлектрических кристаллов, тонких пленок и наноструктур [15-19] и, в отличие от рентгеновской дифракции, позволяет исследовать динамику сегнетоэлектрического параметра порядка.

Несмотря на все преимущества, на данный момент есть лишь несколько работ, в которых метод ГВГ применяется для исследования такого класса материалов, как сегнетоэлектрики-полупроводники [20-23], в которых наблюдение оптического переключения сегнетоэлектрического параметра порядка наиболее вероятно за счет экранирования поляризации фотовозбужденными в зону проводимости носителями заряда.

В данной работе исследовались возбуждение и динамика нелинейно-оптического отклика кристалла сегнетоэлектрика-полупроводника SPS с помощью фемтосекундного лазерного импульса. Было показано, что под воздействием фемтосекундных импульсов в образце происходит изменение интенсивности ГВГ, что может быть связано с экранировкой существующей электрической поляризации. 


\section{2. Экспериментальная установка. Методика эксперимента}

В исследованиях использовался монокристалл гипотиодифосфата олова $\mathrm{Sn}_{2} \mathrm{P}_{2} \mathrm{~S}_{6}$, срезанный по кристаллографической плоскости [010].

Кристалл SPS представляет собой интересный с исследовательской точки зрения, широкозонный полупроводник-сегнетоэлектрик с уникальными нелинейно-оптическими свойствами и широкой полосой пропускания оптического излучения в диапазоне от $530 \mathrm{~nm}$ до $8 \mu \mathrm{m}$. При комнатной температуре кристалл SPS является сегнетоэлектриком с моноклинной точечной группой симметрии $m$ и имеет фазовый переход второго рода в параэлектрическую фазу с точечной группой симметрии $2 / m$ при температуре около $337 \mathrm{~K}$ [24]. Вместе с тем, этот материал проявляет свойства широкозонного полупроводника с запрещенной зоной $E_{g}=2.31 \mathrm{eV}$. Выбор длин волн для эксперимента по схеме накачка-зондирование был сделан на основе результатов, приведенных в работе [25], в которой было показано, что для электромагнитного излучения с энергией фотонов меньше $2 \mathrm{eV}$ коэффициент поглощения в этом материале крайне мал $\left(\leq 3 \mathrm{~cm}^{-1}\right)$, в то время как при энергиях выше $2 \mathrm{eV}$ поглощение быстро возрастает, достигая величин порядка $10^{5} \mathrm{~cm}^{-1}$. Такое поведение характерно для полупроводниковых материалов, так же, как и явление фотопроводимости, которое для SPS изучалось в работах $[25,26]$.

Диаграмма энергетических уровней кристалла SPS приведена в работе [25]. Показано, что зона проводимости состоит из нескольких узких подзон. В работе [27] утверждалось, что благодаря ионам $\mathrm{Sn}^{+}$, играющим роль акцептора, нелегированный SPS является полупроводником p-типа при комнатной температуре.

Для исследований кинетики релаксации второй оптической гармоники в кристалле SPS использовалась методика оптической накачки-зондирования. Схема экспериментальной установки аналогична схеме, приведенной в работе [28]. В качестве источника излучения использовались импульсы, сгенерированные твердотельным лазером на кристалле сапфира, допированного ионами титана. Центральная длина волны излучения такого лазера составляла $800 \mathrm{~nm}$. Далее излучение проходило через усилитель и компрессор так, что в итоге получалась последовательность лазерных импульсов с длительностью каждого импульса 80-100 fs и с частотой повторения $1 \mathrm{kHz}$. После этого излучение разделялось светоделителем между каналом зондирующего излучения и каналом накачки в соотношении интенсивностей $3: 7$.

Спектр луча накачки мог перестраиваться с помощью оптического параметрического усилителя. Излучение накачки с длиной волны $570 \mathrm{~nm}$ (энергия фотона $2.18 \mathrm{eV}$ ), проходя через линию задержки, фокусировалось на поверхность образца. Луч накачки падал на образец параллельно нормали к поверхности образца.

Центральная длина волны зондирующего импульса была выбрана равной $800 \mathrm{~nm}$ (энергия фотона $1.55 \mathrm{eV}$ ).
Поляризация обоих лучей была линейной. Угол падения зондирующего луча составлял 45 градусов. Детектирование происходило на длине волны второй оптической гармоники $(400 \mathrm{~nm})$ с помощью фотоэлектронного умножителя (ФЭУ), сигнал с которого обрабатывался счетчиком фотонов (Stanford research). Для детектирования сигнала только на длине волны ВГ остальные излучения блокировались узкополосными фильтрами. Следует отметить, что даже при сравнительно малых интенсивностях возбуждающего излучения поверхность образца может повреждаться в результате воздействия лазерного излучения. Это может быть связано с эффектом „усталости“, когда каждый возбуждающий импульс создает и оставляет микроскопические дефекты, накопление которых приводит к разрушению образца. Поэтому подбор мощности возбуждающего излучения имеет большое значение для интерпретации результатов эксперимента.

Для возможности нагрева образца в экспериментальную установку был добавлен нагревательный элемент и контроллер, позволяющий изменять и удерживать заданную температуру.

\section{3. Генерация второй оптической гармоники}

3.1. Спектральная зависимость. Для выявления спектральных особенностей кристалла сегнетоэлектрика-полупроводника SPS были исследованы спектральные зависимости генерации второй оптической гармоники, а также люминесценции при двухфотонном возбуждении (ДФЛ) в диапазоне длин волн 730-950 nm. На рис. 1 приведены характерные спектры люминесценции кристалла SPS при длинах волн накачки $800 \mathrm{~nm}$ $(1.55 \mathrm{eV}), 840 \mathrm{~nm}(1.48 \mathrm{eV}), 880 \mathrm{~nm}(1.41 \mathrm{eV})$ и $900 \mathrm{~nm}$ $(1.38 \mathrm{eV})$. Коротковолновые узкие пики соответствуют

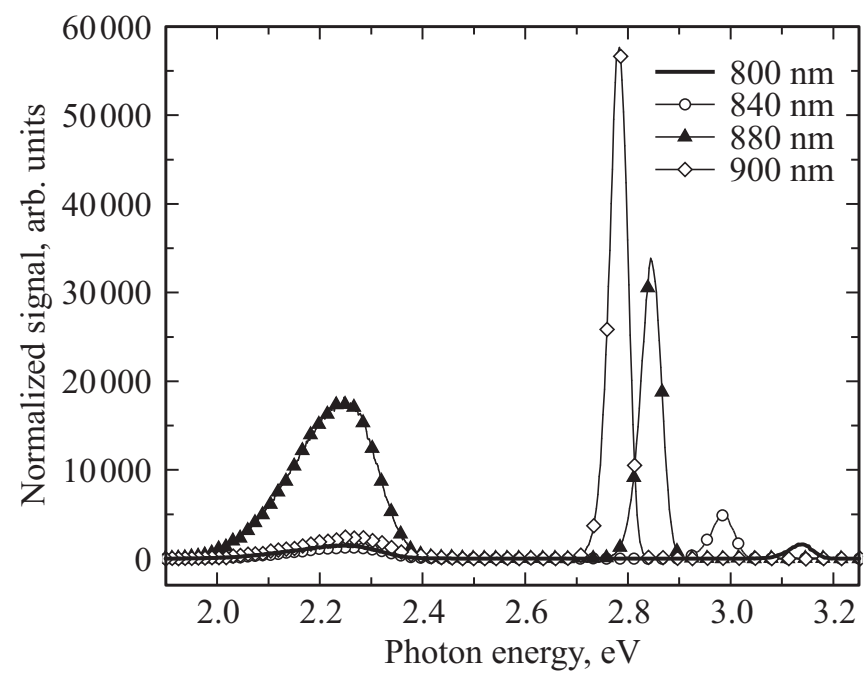

Рис. 1. Спектры люминесценции кристалла SPS при длинах волн накачки 800, 840, 880 и $900 \mathrm{~nm}$. 
генерации излучения на удвоенной частоте, а более широкие пики с центром в области $2.25 \mathrm{eV}(550 \mathrm{~nm})$ отвечают люминесценции при двухфотонном поглощении. При увеличении длины волны накачки от 750 до $950 \mathrm{~nm}$ интенсивность пика ВГ резко возрастает, увеличиваясь примерно на два порядка. Интенсивность пика люминесценции максимальна при длине волны накачки в области $880 \mathrm{~nm}$, а его положение незначительно (в пределах $10 \mathrm{~nm})$ сдвигается в сторону более коротких волн при увеличении длины волны накачки.

3.2. Зависимость интенсивности генерации второй оптической гармоники от температуры. Феноменологически процесс генерации второй оптической гармоники описывается с помощью нелинейной поляризации. Наведение поляризации световым полем описывается материальным уравнением

$$
\mathbf{P}=\mathbf{P}(\mathbf{E}),
$$

которое отражает структуру и свойства среды. Простейшее материальное уравнение нелинейной среды имеет вид

$$
P=\chi^{(1)} E+\chi^{(2)} E^{2}+\chi^{(3)} E^{3}+\ldots+\chi^{(m)} E^{m}+\ldots
$$

Согласно этому уравнению поляризация среды есть нелинейная функция напряженности светового поля $E$.

Выделяя в поляризации среды линейную и нелинейную компоненты, можно записать

$$
\mathbf{P}=\mathbf{P}_{l}+\mathbf{P}_{n l},
$$

где

$$
\begin{gathered}
P_{l}(E)=\chi^{(1)} E \\
P_{n l}(E)=\chi^{(2)} E^{2}+\chi^{(3)} E^{3}+\ldots+\chi^{(m)} E^{m}+\ldots
\end{gathered}
$$

Здесь $\chi^{(m)}$ - нелинейные восприимчивости соответствующего порядка. Коэффициенты $\chi^{(m)}$ при членах разложения (5) являются тензорами $(m+1)$-го порядка. Тогда волновое уравнение, полученное из уравнений Максвелла, будет иметь вид

$$
\operatorname{rot} \operatorname{rot} \mathbf{E}+\frac{1}{c^{2}} \frac{\partial^{2} \mathbf{E}}{\partial t^{2}}+\frac{4 \pi}{c^{2}} \frac{\partial^{2} \mathbf{P}_{l}}{\partial t^{2}}=-\frac{4 \pi}{c^{2}} \frac{\partial^{2} \mathbf{P}_{n l}}{\partial t^{2}}
$$

для нелинейной анизотропной среды и

$$
\Delta \mathbf{E}-\frac{1}{c^{2}} \frac{\partial^{2} \mathbf{E}}{\partial t^{2}}-\frac{4 \pi}{c^{2}} \frac{\partial^{2} \mathbf{P}_{1}}{\partial t^{2}}=\frac{4 \pi}{c^{2}} \frac{\partial^{2} \mathbf{P}_{n l}}{\partial t^{2}}
$$

для нелинейной изотропной среды.

Эти уравнения показывают, что нелинейная поляризация среды является источником новых спектральных компонент поля [29].

Квадратично зависящий от напряженности поля световой волны член разложения (5) будет источником излучения на удвоенной частоте. Пусть в среде, обладающей нелинейной восприимчивостью второго порядка $\chi^{(2)}$ распространяется монохроматическая плоская световая волна

$$
E(r, t)=E_{0} \exp \{-i \omega t+k r\} .
$$

При распространении волны в нелинейной среде будет возбуждаться волна поляризации

$$
P_{n l}(2 \omega)=\chi^{(2)} E_{0}^{2} \exp \{-i 2 \omega t+2 k r\} .
$$

Эта волна на частоте излучения $2 \omega$ и будет источником излучения второй гармоники.

Важным преимуществом методики ГВГ является чувствительность к изменению кристаллической структуры образца в процессе фазового перехода. В частности, при переходе из нецентросиметричной в центросимметричную фазу многократно уменьшается интенсивность генерации второй оптической гармоники. Это обусловлено тем, что для данного типа симметрии генерация второй гармоники в электродипольном приближении, которая записывается как

$$
\mathbf{P}^{D}(2 \omega)=\hat{\chi}^{D(2)} \mathbf{E}(\omega) \mathbf{E}(\omega)
$$

запрещена, так как в центросимметричной среде $\hat{\chi}^{D(2)}=0$.

Для центросимметричной среды механизм генерации нелинейной поляризации является квадрупольным и записывается как

$$
\mathbf{P}^{Q}(2 \omega)=\hat{\chi}^{Q(2)} \mathbf{E}(\omega) \nabla \mathbf{E}(\omega),
$$

поэтому эффективность ГВГ падает на 6-8 порядков $[30,31]$.

В сегнетоэлектрических кристаллах с центросимметричной высокотемпературной фазой компоненты тензора нелинейной восприимчивости $\hat{\chi}$ пропорциональны сегнетоэлектрической поляризации [32]. А так как интенсивность ВГ $I_{2 \omega}$ пропорциональна квадрату напряженности поля ВГ

$$
E_{2 \omega} \propto E^{2}(2 \omega)
$$

то получим связь интенсивности ВГ с сегнетоэлектрической поляризацией в виде:

$$
I_{2 \omega} \propto\left(P^{0}\right)^{2}
$$

На рис. 2 приведена температурная зависимость интенсивности генерации второй оптической гармоники кристалла SPS. Видно, что при увеличении температуры интенсивность генерации гармоники резко спадает, приближаясь к нулю при температурах выше критической. Это свидетельствует о переходе структуры кристалла из сегнетоэлектрической фазы в параэлектрическую, где генерация ВГ запрещена.

Согласно выражению (13), интенсивность второй гармоники пропорциональна квадрату поляризации, которая в сегнетоэлектриках является параметром порядка. 


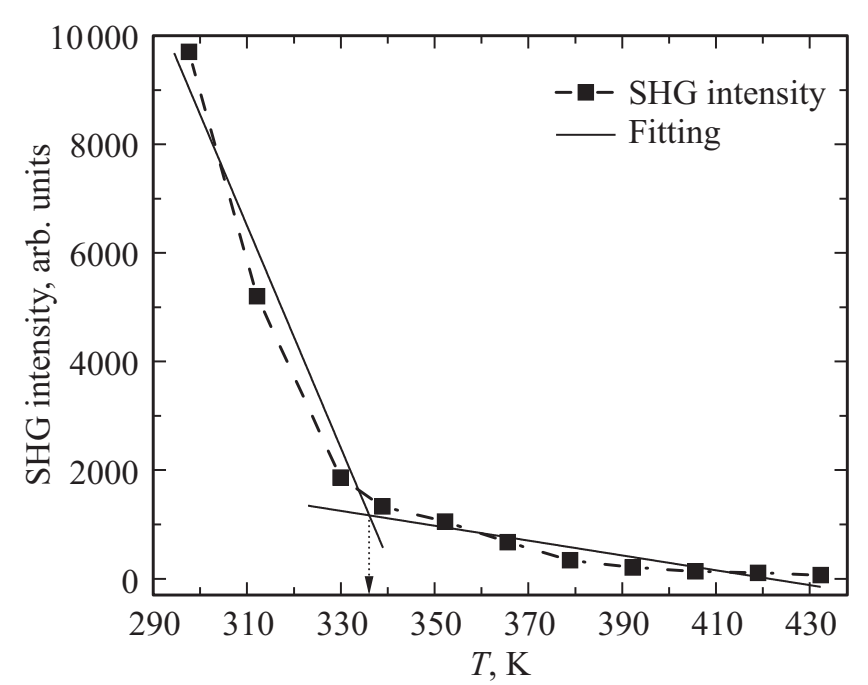

Рис. 2. Зависимость интенсивности генерации второй оптической гармоники в кристалле SPS от температуры нагрева образца.

В рамках теории Ландау, в модели среднего поля зависимость параметра порядка от температуры описывается выражением [33]

$$
P \propto\left(T_{c}-T\right)^{1 / 2}
$$

Таким образом,

$$
I_{2 w} \propto\left(T_{c}-T\right) .
$$

Видно, что зависимость на рис. 2 имеет два ярко выраженных линейных участка. Аппроксимируя эту зависимость двумя прямыми и найдя место их пересечения, определим температуру Кюри равной $336 \mathrm{~K}$, что хорошо согласуется с литературными данными.

Отличие сигнала ВГ от нуля сразу выше температуры Кюри можно объяснить „размыванием“ фазового перехода в результате наличия дефектов в приповерхностном слое и на поверхности кристалла. В этих областях наблюдается слабая генерация ВГ даже выше точки Кюри за счет нарушения центральной симметрии структуры.

\section{4. Фотоиндуцированная динамика генерации второй оптической гармоники}

4.1. Кинетика релаксации нелинейно-оптического отклика. Исследование светоиндуцированной динамики возбуждения и релаксации интенсивности ГВГ при комнатной температуре проводилось при временах задержки между возбуждающим и зондирующим импульсами до $2.5 \mathrm{~ns}$.

Результаты этого эксперимента показаны на рис. 3. Видно, что возбуждение сегнетоэлектрика-полупроводника фотонами с энергией $2.18 \mathrm{eV}$ приводит к резкому изменению интенсивности ГВГ. Изменения происходят на временах порядка длительности импульса. Далее фотоиндуцированный сигнал релаксирует по экспоненциальному закону. Полученные экспериментальные данные были аппроксимированы с помощью функции, основанной на подходе, разработанном ранее для описания аналогичных процессов в прямозонных полупроводниках [34]

$$
\begin{aligned}
y= & A_{\exp }\left(\frac{w^{2}}{\tau_{1}^{2}}-\frac{\tau_{d}}{\tau_{1}}\right)\left[1-\operatorname{erf}\left(\frac{w}{\tau_{1}}-\frac{\tau_{d}}{2 w}\right)\right] \\
& +B_{\exp }\left(\frac{w^{2}}{\tau_{2}^{2}}-\frac{\tau_{d}}{\tau_{1}}\right)\left[1-\operatorname{erf}\left(\frac{w}{\tau_{2}}-\frac{\tau_{d}}{2 w}\right)\right],
\end{aligned}
$$

где $\tau_{d}$ - время задержки между возбуждающим и зондирующим импульсами, $\tau_{1}$ и $\tau_{2}-$ время термализации и рекомбинации носителей заряда соответственно, $A$ и $B$ - константы, $w$ - длительность импульса. Ранее нами было установлено, что в этом материале время термализации $\tau_{1}=1.62 \pm 0.03 \mathrm{ps}$ [13]. С учетом этого в данной работе было установлено, что характерное время рекомбинации электронов из зоны проводимости в валентную зону $\tau_{2} \sim 620 \mathrm{ps}$.

4.2. Температурная зависимостькинетики релаксации нелинейно-оптического отклика. Для того, чтобы понять природу фотоиндуцированных изменений нелинейно-оптического отклика, исследовалась динамика генерации второй оптической гармоники во временном диапазоне до 5 рs при различных температурах. Результаты эксперимента представлены на рис. 4. Из рисунка видно, что при увеличении температуры образца изменяется величина $\Delta I-$ относительное изменение сигнала интенсивности ГВГ, которое определяется как высота „ступеньки“ в сигнале ГВГ между областями $\tau_{d}<0$ и характерными временами

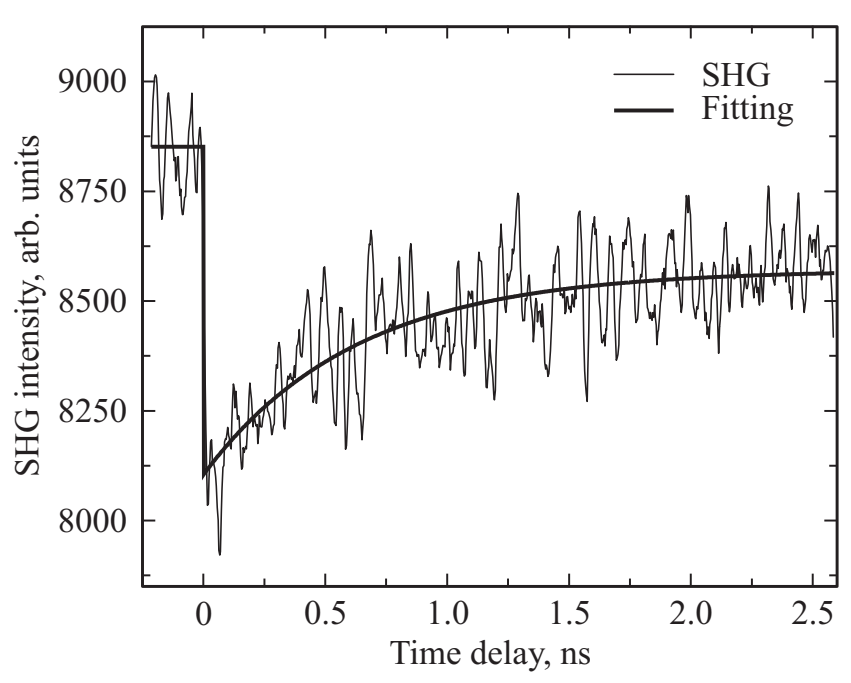

Рис. 3. Зависимость интенсивности генерации второй оптической гармоники от времени задержки между возбуждающим и зондирующим импульсами при комнатной температуре. 


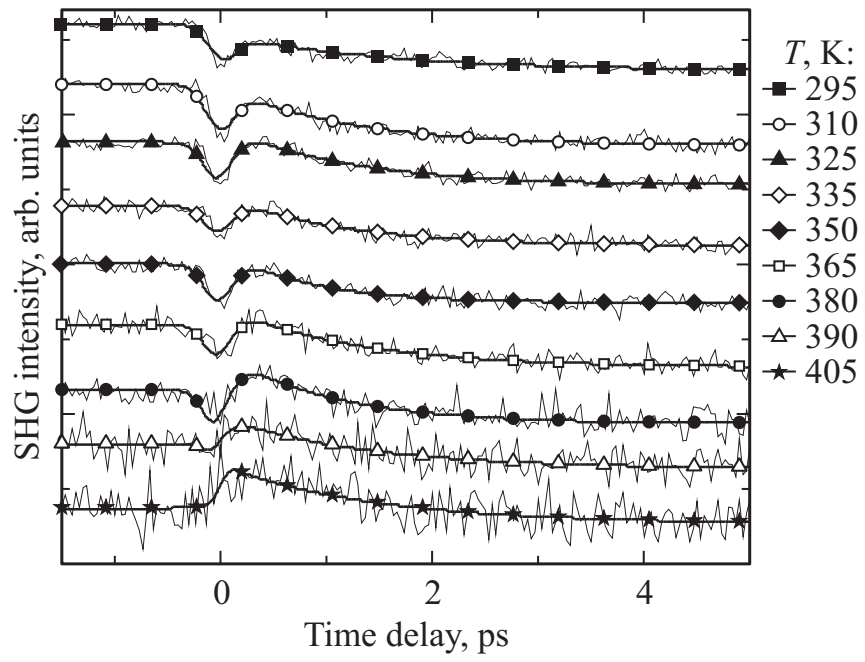

Рис. 4. Зависимость интенсивности генерации второй оптической гармоники от $\tau_{d}-$ времени задержки между возбуждающим и зондирующим импульсами для различных температур. Сигнал ВГ нормирован на интенсивность генерации в области $\tau_{d}<0$. Сплошными линиями показаны экспериментальные данные, линиями с символами - аппроксимация.

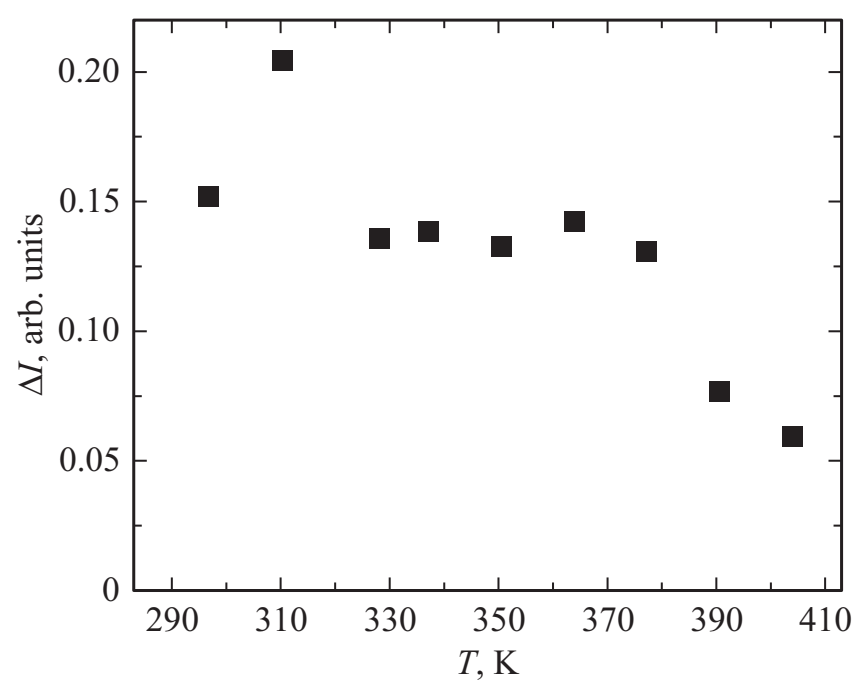

Рис. 5. Зависимость $\Delta I$ от температуры образца.

термализации носителей. Также можно отметить изменение характера временной зависимости в области до 1 ps при температурах выше критической. Этот факт требует дополнительных исследований и в данной работе не рассматривается.

На рис. 5 показана зависимость $\Delta I$ от температуры нагрева образца. Размер символов на рисунке превышает значение погрешности в каждой точке.

Изменение $\Delta I$ пропорционально изменению квадрата сегнетоэлектрической поляризации: $\Delta I \propto \Delta P^{2}$. При нагреве

$$
\Delta P=\frac{d P}{d T} \Delta T
$$

значит

$$
\Delta I \propto \frac{d P^{2}}{d T} \Delta T
$$

$\Delta T$ - изменение температуры и от фактической температуры образца не зависит. $P^{2} \propto\left(T_{c}-T\right)$, а значит $d P^{2} / d T$ также не зависит от температуры образца. Таким образом получаем, что и $\Delta I$ не зависит от температуры.

Из рис. 2 видно, что при нагреве $d P^{2} / d T$ изменяется в точке Кюри.

Таким образом, сравнивая зависимости на рис. 2 и рис. 5 в области фазового перехода, можно сделать вывод о том, что наблюдаемая динамика интенсивности сигнала ГВГ не связана со сверхбыстрым лазерным нагревом кристалла или с эффективным изменением точки фазового перехода, так как это равносильно нагреву/охлаждению образца по отношению к точке Кюри. Наиболее вероятной причиной регистрируемых фотоиндуцированных изменений является экранировка фотоиндуцированными носителями заряда той части электрической поляризации, которая зондируется второй оптической гармоникой. При этом не все фотовозбужденные носители заряда релаксируют. Часть из них захватывается долгоживущими состояниями и создает дефекты, накопление которых приводит к разрушению образца.

\section{5. Заключение}

В работе были исследованы спектральные зависимости генерации второй оптической гармоники, а также люминесценции при двухфотонном возбуждении для кристалла сегнетоэлектрика-полупроводника $\mathrm{Sn}_{2} \mathrm{P}_{2} \mathrm{~S}_{6}$. Установлено, что при увеличении длины волны возбуждающего излучения от 750 до $950 \mathrm{~nm}$ интенсивность ГВГ резко возрастает, увеличиваясь примерно на два порядка, а интенсивность пика люминесценции максимальна при длине волны накачки в области $880 \mathrm{~nm}$.

Исследование релаксации светоиндуцированной динамики генерации второй оптической гармоники при комнатной температуре при временах задержки между возбуждающим и зондирующим импульсами до $2.5 \mathrm{~ns}$ позволило установить, что характерное время рекомбинации электронов из зоны проводимости в валентную зону составляет $\tau_{2} \sim 620 \mathrm{ps}$.

Из характера временных зависимостей интенсивности ГВГ, измеренных при разных температурах, был сделан вывод о том, что воздействие света не приводит с сверхбыстрому нагреву или изменению точки Кюри, а наиболее вероятной причиной наблюдаемых изменений фотоиндуцированной динамики интенсивности ГВГ является экранировка фотоиндуцированными носителями заряда той части электрической поляризации, которая зондируется второй оптической гармоникой. 


\section{Список литературы}

[1] E. Beaurepaire, J.C. Merle, A. Daunois, J.Y. Bigot. Phys. Rev. Lett. 76, 4250 (1996).

[2] G. Ju, A. Nurmikko, R. Farrow, R. Marks, M. Carey, B. Gurney. Phys. Rev. Lett. 82, 3705 (1999).

[3] M. Van Kampen, C. Jozsa, J.T. Kohlhepp, P. LeClair, L. Lagae, W.J.M. De Jonge, B. Koopmans. Phys. Rev. Lett. 88, 227201 (2002).

[4] A.V. Kimel, A. Kirilyuk, P.A. Usachev, R.V. Pisarev, A.M. Balbashov, T. Rasing. Nature 435, 655 (2005).

[5] E.G. Galkina, I.Y. Mikhailov, B.A. Ivanov. JETP Lett. 93, 711 (2011).

[6] A.V. Kimel, A. Kirilyuk, A. Tsvetkov, R.V. Pisarev, T. Rasing. Nature 429, 850 (2004).

[7] C.D. Stanciu, F. Hansteen, A.V. Kimel, A. Kirilyuk, A. Tsukamoto, A. Itoh, T. Rasing. Phys. Rev. Lett. 99, (2007).

[8] K. Vahaplar, A.M. Kalashnikova, A.V. Kimel, D. Hinzke, U. Nowak, R. Chantrell, A. Tsukamoto, A. Itoh, A. Kirilyuk, T. Rasing. Phys. Rev. Lett. 103, 117201 (2009).

[9] S. Mangin, M. Gottwald, C.H. Lambert, D. Steil, V. Uhlír, L. Pang, M. Hehn, S. Alebrand, M. Cinchetti, G. Malinowski, Y. Fainman, M. Aeschlimann, E.E. Fullerton. Nature Mater. 13, 286 (2014).

[10] C-H. Lambert, S. Mangin, B.S.D.C.S. Varaprasad, Y.K. Takahashi, M. Hehn, M. Cinchetti, G. Malinowski, K. Hono, Y. Fainman, M. Aeschlimann, E.E. Fullerton. Science 345, 1337 (2014).

[11] A. Stupakiewicz, K. Szerenos, D. Afanasiev, A. Kirilyuk, A.V. Kimel. Nature 542, 71 (2017).

[12] G.A. Garrett, J.F. Whitaker, A.K. Sood, R. Merlin. Opt. Express 1, 385 (1997).

[13] K.A. Brekhov, K.A. Grishunin, D.V. Afanas'ev, S.V. Semin, N.E. Sherstyuk, G.K. Kitaeva, E.D. Mishina, T. Rasing, A.V. Kimel. JETP Lett. 102, 372 (2015).

[14] S. Grübel, J.A. Johnson, P. Beaud, C. Dornes, A. Ferrer, V. Haborets, L. Huber, T. Huber, A. Kohutych, T. Kubacka, M. Kubli, S.O. Mariager, J. Rittmann, J.I. Saari, Y. Vysochanskii, G. Ingold, S.L. Johnson. arXiv (2016).

[15] T.F. Heinz. Second-Order Nonlinear Optical Effects at Surfaces and Interfaces. In: Nonlinear Surface Electromagnetic Phenomena / Eds H. Ponath, G. Stegeman. North Holland Pub., Амстердам (1991). C. 353-416.

[16] L.V. Simagina, E.D. Mishina, S.V. Semin, N.A. Ilyin, T.R. Volk, R.V. Gainutdinov, L.I. Ivleva. J. Appl. Phys. 110, 52015 (2011).

[17] E.D. Mishina. Ferroelectrics 314, 57 (2005).

[18] E.D. Mishina, N.É. Sherstyuk, V.O. Val'dner, A.V. Mishina, K.A. Vorotilov, V.A. Vasil'ev, A.S. Sigov, M.P. De Santo, E. Cazzanelli, R. Barberi, T. Rasing. Phys. Solid State 48, 1210 (2006).

[19] E.D. Mishina, N.E. Sherstyuk, V.I. Stadnichuk, A.S. Sigov, V.M. Mukhorotov, Y.I. Golovko, A. van Etteger, T. Rasing. Appl. Phys. Lett. 83, 2402 (2003).

[20] A. Anema, A. Grabar, T. Rasing. Ferroelectrics 183, 181 (1996).

[21] К.А. Брехов, К.А. Гришунин, О.В. Самотохин. Фундам. проблемы радиоэлектрон. приборостроения 14, 59 (2014).

[22] К.А. Гришунин, К.А. Брехов, О.В. Самотохин. Росс. технол. журн. 2, 7, 134 (2015).

[23] W.Q. Liao, Y. Zhang, C.L. Hu, J.G. Mao, H.-Y. Ye, P.F. Li, S.D. Huang, R.G. Xiong. Nature Commun. 6, 7338 (2015).
[24] Y.M.V.R.M. Yevych. Condens. Matter Phys. 11, 417 (2008).

[25] R.V. Gamernyk, Y.P. Gnatenko, P.M. Bukivskij, P.A. Skubenko, A.G. Slivka. J. Phys. Condens. Matter 18, 5323 (2006).

[26] A.A. Grabar. Ferroelectrics 192, 155 (1997).

[27] Y.M. Vysochanskii, K. Glukhov, M. Maior, K. Fedyo, A. Kohutych, V. Betsa, I. Pritz, M. Gurzan. Ferroelectrics 418, 124 (2011).

[28] R. Medapalli, I. Razdolski, M. Savoini, A.R. Khorsand, A. Kirilyuk, A.V. Kimel, T. Rasing, A.M. Kalashnikova, A. Tsukamoto, A. Itoh. Phys. Rev. B 86, 54442 (2012).

[29] С.А. Ахманов, С.Ю. Никитин. Физическая оптика, Наука, M. (2004). 654 c.

[30] Н. Бломберген. Нелинейная оптика / Под ред. С.А. Ахманова и Р.В. Хохлова. Мир, М. (1966). 424 с.

[31] R.R. Birss. Symmetry and Magnetism. North Holland Pub., Амстердам (1964). 252 с.

[32] G. Dolino, J. Lajzerowicz, M. Vallade. Phys. Rev. B 2, 2194 (1970).

[33] М. Лайнс, А. Гласс. Сегнетоэлектрики и родственные им материалы / Под ред. В.В. Леманова и Г.А. Смоленского, Мир, М. (1981). 736 c.

[34] A. Kimel, F. Bentivegna, V. Gridnev, V. Pavlov, R. Pisarev, T. Rasing. Phys. Rev. B 63, 1 (2001). 